\title{
POSITIVE SOLUTIONS FOR SINGULAR DISCRETE BOUNDARY VALUE PROBLEMS
}

\author{
MARIELLA CECCHI, ZUZANA DOŠLÁ, AND MAURO MARINI
}

Received 10 December 2002

We study the existence of zero-convergent solutions for the second-order nonlinear difference equation $\Delta\left(a_{n} \Phi_{p}\left(\Delta x_{n}\right)\right)=g\left(n, x_{n+1}\right)$, where $\Phi_{p}(u)=|u|^{p-2} u, p>1,\left\{a_{n}\right\}$ is a positive real sequence for $n \geq 1$, and $g$ is a positive continuous function on $\mathbb{N} \times\left(0, u_{0}\right)$, $0<u_{0} \leq \infty$. The effects of singular nonlinearities and of the forcing term are treated as well.

\section{Introduction}

In this paper, we study decaying nonoscillatory solutions of the second-order difference equation

$$
\Delta\left(a_{n} \Phi_{p}\left(\Delta x_{n}\right)\right)=g\left(n, x_{n+1}\right)
$$

where $\Delta$ is the forward difference operator $\Delta x_{n}=x_{n+1}-x_{n},\left\{a_{n}\right\}$ is a positive real sequence for $n \geq 1, g$ is a positive continuous function on $\mathbb{N} \times\left(0, u_{0}\right), 0<u_{0} \leq \infty$, and $\Phi_{p}(u)=|u|^{p-2} u$ with $p>1$. The left-hand side in (1.1) is the one-dimensional discrete analogue of the $p$-Laplacian $u \rightarrow \operatorname{div}|\nabla u|^{p-2} \nabla u$ that appears in searching for radial solutions of nonlinear partial equations modelling various reaction-diffusion problems (see, e.g., [8]).

Observe that our assumptions on $g$ allow us to consider the "singular case," that is, the case in which the nonlinearity $g$ is unbounded with respect to the second variable in a right neighborhood of zero. From this point of view, a typical example is the nonlinear equation

$$
\Delta\left(a_{n} \Phi_{p}\left(\Delta x_{n}\right)\right)=b_{n}\left[\Phi_{q}\left(x_{n+1}\right)\right]^{-1}+r_{n}
$$

where $\left\{b_{n}\right\}$ and $\left\{r_{n}\right\}$ are real sequences with $b_{n} \geq 0, r_{n} \geq 0$, and $b_{n}+r_{n}>0$ for $n \geq 1$ and $q>1$. 
Equation (1.1) includes also the "regular case" with the forcing term

$$
\Delta\left(a_{n} \Phi_{p}\left(\Delta x_{n}\right)\right)=b_{n} \Phi_{q}\left(x_{n+1}\right)+r_{n}
$$

Positive decreasing solutions of (1.3) when $b_{n}>0$ and $r_{n} \equiv 0$ for $n \geq 1$ have been investigated in $[5,6]$.

Our aim is to study the existence of decaying solutions of (1.1), that is, positive solutions $\left\{x_{n}\right\}$ of (1.1) approaching zero as $n \rightarrow \infty$, in view of their crucial role in a variety of physical applications (see, e.g., [8]). By using a topological approach, we study mainly the effects of singular nonlinearities and those of the forcing term. Our results are also motivated also by some recent effects stated in the continuous case, see, for example, $[1,4,9,12]$ and the references therein. Our results complement the ones in $[10,11]$, where the existence of unbounded solutions of (1.1) is considered under the assumption $b_{n}<0$. Finally, we recall that boundary value problems for equations in a discrete interval $\left[1, N_{0}\right]$ with singular nonlinear term in this interval have been considered recently in $[2,3]$.

\section{Notation and preliminaries}

A solution $\left\{x_{n}\right\}$ of (1.1) is said to be a decaying solution if $x_{n}>0, \Delta x_{n}<0$ eventually, and $\lim _{n} x_{n}=0$. According to the asymptotic behavior of the quasidifference

$$
x_{n}^{[1]}=a_{n} \Phi_{p}\left(\Delta x_{n}\right),
$$

a decaying solution $\left\{x_{n}\right\}$ of (1.1) is called a regularly decaying solution or a strongly decaying solution according to $\lim _{n} x_{n}^{[1]}<0$ or $\lim _{n} x_{n}^{[1]}=0$, respectively. It is easy to show that every decaying solution $\left\{x_{n}\right\}$ of (1.1) satisfies, for every $n \geq 1$,

$$
x_{n}>0, \quad \Delta x_{n}<0 .
$$

Indeed, assume that (2.2) is verified for $n \geq N>1$ and suppose there exists $n_{0}<N$ such that $\Delta x_{n_{0}} \geq 0, \Delta x_{i}<0, x_{i}>0$, for $i=n_{0}+1, \ldots, N$. From (1.1) we obtain

$$
x_{N}^{[1]}=x_{n_{0}}^{[1]}+\sum_{i=n_{0}}^{N-1} g\left(i, x_{i+1}\right)>0
$$

that implies $\Delta x_{N}>0$, that is, a contradiction.

The set of decaying solutions will be denoted by $\mathbb{D}$ and those of regularly decaying solutions and strongly decaying solutions by $\mathbb{D}_{R}$ and $\mathbb{D}_{S}$, respectively. Clearly, $\mathbb{D}=\mathbb{D}_{R} \cup \mathbb{D}_{S}$ and

$$
\begin{aligned}
& \mathbb{D}_{R}=\left\{\left\{x_{n}\right\} \text { solution of }(1.1): x_{n}>0, \Delta x_{n}<0, \lim _{n} x_{n}=0, \lim _{n} x_{n}^{[1]}<0\right\}, \\
& \mathbb{D}_{S}=\left\{\left\{x_{n}\right\} \text { solution of }(1.1): x_{n}>0, \Delta x_{n}<0, \lim _{n} x_{n}=0, \lim _{n} x_{n}^{[1]}=0\right\} .
\end{aligned}
$$


Some notations are in order. Denote

$$
Y_{a}=\lim _{m \rightarrow \infty} \sum_{n=1}^{m} \frac{1}{\Phi_{p^{*}}\left(a_{n}\right)},
$$

where $p^{*}$ denotes the conjugate number of $p$, that is, $p^{*}=p /(p-1)$ or $1 / p+1 / p^{*}=1$.

When $Y_{a}<\infty$, denote by $\left\{A_{n}\right\}$ the sequence given by

$$
A_{n}=\sum_{k=n}^{\infty} \frac{1}{\Phi_{p^{*}}\left(a_{k}\right)} .
$$

We close this section by recalling the following lemma which is the discrete analogue of the Lebesgue dominated convergence theorem and plays an important role in proving topological properties of certain operators associated to the problem of existence of decaying solutions of (1.1).

Lemma 2.1. Let $\left\{\alpha_{i, k}\right\}$ be a double real sequence, $\alpha_{i, k} \geq 0$, for $i, k \in \mathbb{N}$. Assume that the series $\sum_{k=1}^{\infty} \alpha_{i, k}$ totally converges, that is, there exists a sequence $\left\{\beta_{k}\right\}$ such that $\alpha_{i, k} \leq \beta_{k}$, $\sum_{k=1}^{\infty} \beta_{k}<\infty$, and let $\lim _{i \rightarrow \infty} \alpha_{i, k}=\varrho_{k}$ for every $k \in \mathbb{N}$. Then the series $\sum_{k=1}^{\infty} \varrho_{k}$ converges and

$$
\lim _{i \rightarrow \infty} \sum_{k=1}^{\infty} \alpha_{i, k}=\sum_{k=1}^{\infty} \varrho_{k} .
$$

\section{Regularly decaying solutions}

In this section, we study the existence of solutions in the class $\mathbb{D}_{R}$. We start with a necessary condition. The following proposition holds.

Proposition 3.1. If $\mathbb{D}_{R} \neq \varnothing$, then $Y_{a}<\infty$.

Proof. Let $x=\left\{x_{n}\right\}$ be a solution of (1.1) in the class $\mathbb{D}_{R}$. Because $\left\{x_{n}^{[1]}\right\}$ is negative increasing and $\lim _{n} x_{n}^{[1]}=x_{\infty}^{[1]}<0$, it holds that

$$
a_{n} \Phi_{p}\left(\Delta x_{n}\right)<x_{\infty}^{[1]}
$$

This implies, for $n<N$,

$$
\Phi_{p^{*}}\left(\left|x_{\infty}^{[1]}\right|\right) \sum_{j=n}^{N-1} \Phi_{p^{*}}\left(\frac{1}{a_{j}}\right) \leq x_{n}-x_{N}
$$

that gives the assertion as $N \rightarrow \infty$.

Remark 3.2. For any solution $\left\{x_{n}\right\} \in \mathbb{D}_{R}$, it holds that $a_{n} \Phi_{p}\left(\Delta x_{n}\right) \geq x_{1}^{[1]}$. Hence, from (3.2), we obtain the following upper and lower bounds:

$$
-\Phi_{p^{*}}\left(x_{\infty}^{[1]}\right) A_{n} \leq x_{n} \leq-\Phi_{p^{*}}\left(x_{1}^{[1]}\right) A_{n} .
$$


274 Positive solutions for BVPs

In addition, regularly decaying solutions $\left\{x_{n}\right\}$ are asymptotic to the sequence (2.6), that is,

$$
\lim _{n} \frac{x_{n}}{A_{n}}=c_{x}, \quad 0<c_{x}<\infty,
$$

where $\Phi_{p}\left(c_{x}\right)=\left|x_{\infty}^{[1]}\right|$, as the Stolze theorem yields.

Assumption $Y_{a}<\infty$ is not sufficient for the existence of solutions in the class $\mathbb{D}_{R}$ as the following example shows.

Example 3.3. Consider the equation

$$
\Delta\left(n^{2} \Phi_{p}\left(\Delta x_{n}\right)\right)=\frac{1}{x_{n+1}} .
$$

Let $\left\{x_{n}\right\}$ be a solution of $(3.5)$ in the class $\mathbb{D}_{R}$ and let $n_{0} \geq 1$ such that $x_{n+1}<1$ for $n>n_{0}$. Hence, for $n>n_{0}$,

$$
\Delta\left(n^{2} \Phi_{p}\left(\Delta x_{n}\right)\right)>1
$$

or

$$
x_{n+1}^{[1]}>x_{n_{0}}^{[1]}+n-n_{0}
$$

that gives a contradiction as $n \rightarrow \infty$.

The following theorem holds.

Theorem 3.4. Assume the following conditions:

(i) $Y_{a}<\infty$;

(ii) there exists a continuous function $F: \mathbb{N} \times(0, \delta] \rightarrow(0, \infty), \delta<u_{0}$, monotone with respect to the second variable such that for $(n, v) \in \mathbb{N} \times(0, \delta]$,

$$
\begin{aligned}
& g(n, v) \leq F(n, v), \\
& \sum_{n=1}^{\infty} F\left(n, A_{n+1}\right)<\infty .
\end{aligned}
$$

Then (1.1) has solutions in the class $\mathbb{D}_{R}$. More precisely, for every $c \geq 1$, there exists a positive solution $\left\{x_{n}\right\}$ such that

$$
\lim _{n} \frac{x_{n}}{A_{n}}=c
$$

where $\Phi_{p}(c)=\lim _{n}\left|x_{n}^{[1]}\right|$.

Proof. First, we prove the statement for $F$ nonincreasing. Choose $n_{0} \geq 1$ such that

$$
\begin{gathered}
\Phi_{p^{*}}(2) A_{n_{0}}<\delta, \\
\sum_{n=n_{0}}^{\infty} F\left(n, A_{n+1}\right)<1 .
\end{gathered}
$$


Denote by $\ell_{n_{0}}^{\infty}$ the Banach space of all bounded sequences defined for $n \geq n_{0}$ and endowed with the topology of supremum norm. Let $\Omega$ be the nonempty subset of $\ell_{n_{0}}^{\infty}$ given by

$$
\Omega=\left\{\left\{u_{n}\right\} \in \ell_{n_{0}}^{\infty}: A_{n} \leq u_{n} \leq \Phi_{p^{*}}(2) A_{n}\right\} .
$$

Clearly, $\Omega$ is a bounded, closed, and convex subset of $\ell_{n_{0}}^{\infty}$. We define the mapping $T: \Omega \rightarrow$ $\ell_{n_{0}}^{\infty}$ by

$$
w_{n}=\sum_{j=n}^{\infty} \Phi_{p^{*}}\left(\frac{1}{a_{j}}\right) \Phi_{p^{*}}\left(1+\sum_{i=j}^{\infty} g\left(i, u_{i+1}\right)\right) .
$$

We prove that $T$ satisfies the hypotheses of Schauder fixed-point theorem.

(a) The mapping $T$ maps $\Omega$ into itself. Obviously, $A_{n} \leq w_{n}$. Conditions (ii) and (3.12) imply

$$
\sum_{j=n_{0}}^{\infty} g\left(j, u_{j+1}\right) \leq \sum_{j=n_{0}}^{\infty} F\left(j, u_{j+1}\right) \leq \sum_{j=n_{0}}^{\infty} F\left(j, A_{j+1}\right) \leq 1,
$$

and taking into account (3.14) and monotonicity of $\Phi_{p^{*}}$, we have

$$
w_{n} \leq \sum_{j=n}^{\infty} \Phi_{p^{*}}\left(\frac{2}{a_{j}}\right)=\Phi_{p^{*}}(2) A_{n} .
$$

(b) The mapping $T$ is continuous in $\Omega$. Let $\left\{U^{(i)}\right\}$ be a sequence in $\Omega$ converging to $U$ in $\ell_{n_{0}}^{\infty}$. Because $\Omega$ is closed, $U \in \Omega$. Let $U^{(i)}=\left\{u_{n}^{(i)}\right\}, U=\left\{u_{n}\right\}$ and $W^{(i)}=T\left(U^{(i)}\right)=$ $\left\{w_{n}^{(i)}\right\}, W=T(U)=\left\{w_{n}\right\}$. It holds for every integer $n \geq n_{0}$ that

$$
\begin{aligned}
& \left\|T\left(U^{(i)}\right)-T(U)\right\| \\
& \quad=\sup _{n \geq n_{0}}\left|w_{n}^{(i)}-w_{n}\right| \\
& \quad \leq \sup _{n \geq n_{0}} \sum_{k=n}^{\infty} \Phi_{p^{*}}\left(\frac{1}{a_{k}}\right)\left|\Phi_{p^{*}}\left(1+\sum_{j=k}^{\infty} g\left(j, u_{j+1}^{(i)}\right)\right)-\Phi_{p^{*}}\left(1+\sum_{j=k}^{\infty} g\left(j, u_{j+1}\right)\right)\right| \\
& \quad \leq \sum_{k=n_{0}}^{\infty} \alpha_{i, k},
\end{aligned}
$$

where

$$
\alpha_{i, k}=\Phi_{p^{*}}\left(\frac{1}{a_{k}}\right)\left|\Phi_{p^{*}}\left(1+\sum_{j=k}^{\infty} g\left(j, u_{j+1}^{(i)}\right)\right)-\Phi_{p^{*}}\left(1+\sum_{j=k}^{\infty} g\left(j, u_{j+1}\right)\right)\right| .
$$

From the continuity of $g$, we obtain

$$
\lim _{i} g\left(j, u_{j+1}^{(i)}\right)=g\left(j, u_{j+1}\right) \quad \text { for } j \geq n_{0},
$$


and, in view of (ii) and the fact that $U^{(i)} \in \Omega$,

$$
\left|g\left(j, u_{j+1}^{(i)}\right)\right| \leq F\left(j, A_{j+1}\right) .
$$

Then the series $\sum_{j=k}^{\infty} g\left(j, u_{j+1}^{(i)}\right)$ is totally convergent and, by Lemma 2.1,

$$
\lim _{i} \Phi_{p^{*}}\left(1+\sum_{j=k}^{\infty} g\left(j, u_{j+1}^{(i)}\right)\right)=\Phi_{p^{*}}\left(1+\sum_{j=k}^{\infty} g\left(j, u_{j+1}\right)\right),
$$

that is,

$$
\lim _{i} \alpha_{i, k}=0 \quad \text { for every } k \geq n_{0} .
$$

In addition, using (3.12), we find

$$
\begin{aligned}
\alpha_{i, k} & \leq\left(\Phi_{p^{*}}\left(\frac{1}{a_{k}}\right)\right)\left(\Phi_{p^{*}}\left(1+\sum_{j=k}^{\infty} F\left(j, u_{j+1}^{(i)}\right)\right)+\Phi_{p^{*}}\left(1+\sum_{j=k}^{\infty} F\left(j, u_{j+1}\right)\right)\right) \\
& \leq 2\left(\Phi_{p^{*}}\left(\frac{1}{a_{k}}\right)\right) \Phi_{p^{*}}\left(1+\sum_{j=k}^{\infty} F\left(j, A_{j+1}\right)\right) \leq 2 \Phi_{p^{*}}(2)\left(\Phi_{p^{*}}\left(\frac{1}{a_{k}}\right)\right) .
\end{aligned}
$$

Since $Y_{a}<\infty$, the series $\sum_{k=n_{0}}^{\infty} \alpha_{i, k}$ is totally convergent. Applying again Lemma 2.1, it follows from (3.17) and (3.22) that

$$
\lim _{i}\left\|T\left(U^{(i)}\right)-T(U)\right\| \leq \lim _{i} \sum_{k=n_{0}}^{\infty} \alpha_{i, k}=\sum_{k=n_{0}}^{\infty}\left[\lim _{i} \alpha_{i, k}\right]=0 .
$$

Hence, $T$ is continuous in $\Omega$.

(c) The set $T(\Omega)$ is relatively compact. By a result in [7, Theorem 3.3], it is sufficient to prove that $T(\Omega)$ is uniformly Cauchy in the topology of $\ell_{n_{0}}^{\infty}$, that is, for every $\varepsilon>0$, there exists an integer $n_{\varepsilon} \geq n_{0}$ such that $\left|w_{m_{1}}-w_{m_{2}}\right|<\varepsilon$ whenever $m_{1}, m_{2}>\varepsilon$ for every $W=\left\{w_{n}\right\} \in T(\Omega)$. Let $W=T(U), U=\left\{u_{n}\right\}$, and, without loss of generality, assume $m_{1}<m_{2}$. From (3.14), we obtain

$$
\begin{aligned}
\left|w_{m_{1}}-w_{m_{2}}\right| & =\left|\sum_{j=m_{1}}^{m_{2}-1} \Phi_{p^{*}}\left(\frac{1}{a_{j}}\right) \Phi_{p^{*}}\left(1+\sum_{i=j}^{\infty} g\left(i, u_{i+1}\right)\right)\right| \\
& \leq\left|\sum_{j=m_{1}}^{m_{2}-1} \Phi_{p^{*}}\left(\frac{1}{a_{j}}\right) \Phi_{p^{*}}\left(1+\sum_{i=j}^{\infty} F\left(i, A_{i+1}\right)\right)\right| \\
& \leq \Phi_{p^{*}}(2) \sum_{j=m_{1}}^{m_{2}-1} \Phi_{p^{*}}\left(\frac{1}{a_{j}}\right),
\end{aligned}
$$

and the Cauchy criterion gives the relative compactness of $T(\Omega)$. 
Hence, by Schauder fixed-point theorem, there exists $\left\{x_{n}\right\} \in \Omega$ such that $x_{n}=T\left(x_{n}\right)$ or, from (3.14),

$$
x_{n}=\sum_{j=n}^{\infty} \Phi_{p^{*}}\left[\frac{1}{a_{j}}\left(1+\sum_{i=j}^{\infty} g\left(i, x_{i+1}\right)\right)\right] .
$$

One can easily check that $\left\{x_{n}\right\}$ is a solution of (1.1) with $\Delta x_{n}<0, \lim _{n} x_{n}=0$, and $\lim _{n} x_{n}^{[1]}=-1$, and so $\left\{x_{n}\right\} \in \mathbb{D}_{R}$. Clearly, in view of Remark 3.2, $\left\{x_{n}\right\}$ satisfies (3.10) with $c=1$.

To obtain the existence of a positive solution $\left\{x_{n}\right\}$ such that $\lim _{n}\left[x_{n} / A_{n}\right]=c>1$, it is sufficient to observe that (3.9) and monotonicity of $F$ imply that the series

$$
\sum_{n=1}^{\infty} F\left(n, \lambda A_{n+1}\right)
$$

is convergent for any $\lambda \geq 1$. Now, the assertion follows by considering in the subset

$$
\Omega_{\lambda}=\left\{\left\{u_{n}\right\} \in \ell_{n_{0}}^{\infty}: \Phi_{p^{*}}(\lambda) A_{n} \leq u_{n} \leq \Phi_{p^{*}}(2 \lambda) A_{n}\right\}
$$

the operator $T:\left\{u_{n}\right\} \rightarrow\left\{w_{n}\right\}$ given by

$$
w_{n}=\sum_{j=n}^{\infty} \Phi_{p^{*}}\left(\frac{1}{a_{j}}\right) \Phi_{p^{*}}\left(\lambda+\sum_{i=j}^{\infty} g\left(i, u_{i+1}\right)\right)
$$

and using an analogous argument as above.

In case $F$ is nondecreasing on $(0, \delta]$, the proof is quite similar with some minor changes. It is sufficient to consider the subset $\Omega$ and the operator $T$ as follows:

$$
\begin{aligned}
\Omega & =\left\{\left\{u_{n}\right\} \in \ell_{n_{0}}^{\infty}: \frac{1}{2} A_{n} \leq u_{n} \leq A_{n}\right\}, \\
w_{n} & =\sum_{j=n}^{\infty} \Phi_{p^{*}}\left(\frac{1}{a_{j}}\left(\frac{1}{2}+\sum_{i=j}^{\infty} g\left(i, u_{i+1}\right)\right)\right),
\end{aligned}
$$

where $n_{0}$ is chosen such that

$$
\sum_{n=n_{0}}^{\infty} F\left(n, A_{n+1}\right)<\frac{1}{2}
$$

The details are left to the reader.

Remark 3.5. The existence of regularly decaying solutions $\left\{x_{n}\right\}$ satisfying (3.10) for $c \in$ $(0,1)$ is guaranteed by the condition

$$
\sum_{n=1}^{\infty} F\left(n, \Phi_{p^{*}}(c) A_{n+1}\right)<\infty
$$

instead of (3.9) and can be proved using an analogous argument as given in the proof of Theorem 3.4. 
For the special case of (1.2), assumption (ii) of Theorem 3.4 becomes

$$
\sum_{n=1}^{\infty} b_{n}\left[\Phi_{q}\left(A_{n+1}\right)\right]^{-1}<\infty, \quad \sum_{n=1}^{\infty} r_{n}<\infty .
$$

In this case, by applying Theorem 3.4 to (1.2), for every $c>0$, we obtain the existence of solutions satisfying (3.10). In addition, for (1.2), conditions $Y_{a}<\infty$ and (3.33) become also necessary for the existence in $\mathbb{D}_{R}$ as the following result shows.

Corollary 3.6. Equation (1.2) has solutions in the class $\mathbb{D}_{R}$ if and only if $Y_{a}<\infty$ and (3.33) hold.

Proof. In view of Proposition 3.1 and Theorem 3.4, it is sufficient to prove that if $\mathbb{D}_{R} \neq \varnothing$, then (3.33) is verified. Let $\left\{x_{n}\right\}$ be a solution of (1.2) in the class $\mathbb{D}_{R}$. By the summation of (1.2) from $n$ to $N-1$ and taking into account (3.3), we have

$$
\begin{aligned}
-x_{n}^{[1]} & =-x_{N}^{[1]}+\sum_{j=n}^{N-1} b_{j}\left[\Phi_{q}\left(x_{j+1}\right)\right]^{-1}+\sum_{j=n}^{N-1} r_{j} \\
& >\lambda \sum_{j=n}^{N} b_{j}\left[\Phi_{q}\left(A_{j+1}\right)\right]^{-1}+\sum_{j=n}^{N} r_{j},
\end{aligned}
$$

where $\lambda=\left[\Phi_{q}\left[\Phi_{p^{*}}\left(-x_{1}^{[1]}\right)\right]\right]^{-1}$. As $N \rightarrow \infty$, we obtain the assertion.

Theorem 3.4 is applicable even if the nonlinearity $g$ is bounded with respect to the dependent variable in a right neighborhood of zero, that is, the boundary value problem is "regular." In such a case, assumption (ii) of Theorem 3.4 can be simplified.

Corollary 3.7. If $Y_{a}<\infty$ and

$$
\sum_{n=1}^{\infty} b_{n} \Phi_{q}\left(A_{n+1}\right)<\infty, \quad \sum_{n=1}^{\infty} r_{n}<\infty,
$$

then (1.3) has solutions in the class $\mathbb{D}_{R}$. More precisely, for every $c>0$, there exists a positive solution $\left\{x_{n}\right\}$ such that (3.10) is verified with $\Phi_{p}(c)=\lim _{n}\left|x_{n}^{[1]}\right|$.

Proof. The assertion follows from Theorem 3.4 and Remark 3.5 by choosing $F(n, v)=$ $b_{n} \Phi_{q}(v)+r_{n}$.

\section{Strongly decaying solutions}

Here we study the existence of solutions in the class $\mathbb{D}_{S}$ for equations with possible singular nonlinearity. More precisely, in this section, we will assume that $g$ satisfies the condition

$$
\inf _{v \in(0, \delta]} g(i, v)=m_{i}>0
$$

for infinitely many $i$, where $\delta$ is a positive constant, $\delta<u_{0}$. The following necessary conditions hold. 
Proposition 4.1. If $\mathbb{D}_{S} \neq \varnothing$, then

$$
\begin{gathered}
\sum_{n=1}^{\infty} m_{n}<\infty, \\
\sum_{j=1}^{\infty} \Phi_{p^{*}}\left(\frac{1}{a_{j}} \sum_{i=j}^{\infty} m_{i}\right)<\infty,
\end{gathered}
$$

where $m_{j}$ is given in (4.1).

Proof. Let $\left\{x_{n}\right\}$ be a solution of (1.1) in the class $\mathbb{D}_{S}$. Without loss of generality, we can assume $x_{n}<\delta$ for $n \geq 1$. Hence,

$$
g\left(i, x_{i+1}\right) \geq \inf _{v \in(0, \delta]} g(i, v)=m_{i}
$$

By summing (1.1) from $n$ to $\infty$, we obtain

$$
-x_{n}^{[1]}=\sum_{i=n}^{\infty} g\left(i, x_{i+1}\right) \geq \sum_{i=n}^{\infty} m_{i}
$$

that implies (4.2). By summing again from $n$ to $\infty$, we have

$$
x_{n} \geq \sum_{i=n}^{\infty} \Phi_{p^{*}}\left(\frac{1}{a_{j}} \sum_{i=j}^{\infty} m_{i}\right)
$$

and so (4.3) is proved.

Remark 4.2. Because

$$
\sum_{j=1}^{N} \Phi_{p^{*}}\left(\frac{1}{a_{j}} \sum_{i=j}^{N} m_{j}\right) \geq \Phi_{p^{*}}\left(\frac{1}{a_{1}} \sum_{i=1}^{N} m_{j}\right)=\Phi_{p^{*}}\left(\frac{1}{a_{1}}\right) \Phi_{p^{*}}\left(\sum_{i=1}^{N} m_{j}\right),
$$

condition (4.3) implies (4.2).

A sufficient criterion for existence in $\mathbb{D}_{S}$ is given by the following theorem.

Theorem 4.3. Assume (4.1) and (4.3). If there exists a continuous function $F: \mathbb{N} \times(0, \delta] \rightarrow$ $(0, \infty), 0<\delta<u_{0}$, nonincreasing with respect to the second variable such that, for $(n, v) \in$ $\mathbb{N} \times(0, \delta]$,

$$
\begin{gathered}
g(n, v) \leq F(n, v) \\
\sum_{n=1}^{\infty} \Phi_{p^{*}}\left[\frac{1}{a_{n}} \sum_{j=n}^{\infty} F\left(j, B_{j+1}\right)\right]<\infty
\end{gathered}
$$

where

$$
B_{n}=\sum_{j=n}^{\infty} \Phi_{p^{*}}\left(\frac{1}{a_{j}} \sum_{i=j}^{\infty} m_{i}\right)
$$

then (1.1) has solutions in the class $\mathbb{D}_{S}$. 
Proof. Choose $n_{0} \geq 1$ such that

$$
B_{n_{0}}<\delta, \quad \sum_{n=n_{0}}^{\infty} \Phi_{p^{*}}\left[\frac{1}{a_{n}} \sum_{j=n}^{\infty} F\left(j, B_{j+1}\right)\right]<\delta .
$$

Let $\Omega$ be the subset of $\ell_{n_{0}}^{\infty}$ given by

$$
\Omega=\left\{\left\{u_{n}\right\} \in \ell_{n_{0}}^{\infty}: B_{n} \leq u_{n} \leq \delta\right\} .
$$

In view of (4.1), it holds that $B_{n}>0$. In addition, because $\left\{B_{n}\right\}$ is nonincreasing, from (4.10) the set $\Omega$ is nonempty. Clearly, $\Omega$ is bounded, closed, and convex in $\ell_{n_{0}}^{\infty}$. We define the mapping $T: \Omega \rightarrow \ell_{n_{0}}^{\infty}$ by

$$
w_{n}=\sum_{j=n}^{\infty} \Phi_{p^{*}}\left[\frac{1}{a_{n}} \sum_{j=n}^{\infty} g\left(j, u_{j+1}\right)\right] .
$$

Because

$$
g\left(j, u_{j+1}\right) \geq \inf _{v \in(0, \delta]} g(j, v)=m_{j}
$$

we have

$$
w_{n} \geq \sum_{j=n}^{\infty} \Phi_{p^{*}}\left[\frac{1}{a_{n}} \sum_{j=n}^{\infty} m_{j}\right]=B_{n} .
$$

In addition, it holds for $j \geq n_{0}$ that

$$
\sum_{j=n}^{\infty} g\left(j, u_{j+1}\right) \leq \sum_{j=n}^{\infty} F\left(j, u_{j+1}\right) \leq \sum_{j=n}^{\infty} F\left(j, B_{j+1}\right)
$$

or, in view of (4.10),

$$
w_{n} \leq \sum_{j=n}^{\infty} \Phi_{p^{*}}\left[\frac{1}{a_{n}} \sum_{j=n}^{\infty} F\left(j, B_{j+1}\right)\right]<\delta .
$$

Thus, $T(\Omega) \subseteq \Omega$. The continuity of $T$ in $\Omega$ and the compactness of $\overline{T(\Omega)}$ follow by using a similar argument as in the proof of Theorem 3.4. Hence, by applying the Schauder fixedpoint theorem, we obtain the existence of a fixed point $\left\{x_{n}\right\}$ of $T$. Clearly,

$$
x_{n}=\sum_{j=n}^{\infty} \Phi_{p^{*}}\left(\frac{1}{a_{j}} \sum_{i=j}^{\infty} g\left(i, x_{i+1}\right)\right),
$$

and so $\left\{x_{n}\right\} \in \mathbb{D}_{S}$.

For the special case of singular equation (1.2) with $r_{n}=0$ for $n \in \mathbb{N}$, Theorem 4.3 yields the following result. 
Corollary 4.4. Consider the equation

$$
\Delta\left(a_{n} \Phi_{p}\left(\Delta x_{n}\right)\right)=b_{n}\left[\Phi_{q}\left(x_{n+1}\right)\right]^{-1}
$$

with $b_{n}>0$ for infinitely many $n$. Assume

$$
\lim _{m \rightarrow \infty} \sum_{n=1}^{m} \Phi_{p^{*}}\left(\frac{1}{a_{n}} \sum_{k=n}^{m} b_{k}\right)<\infty
$$

and denote

$$
\beta_{n}=\sum_{i=n}^{\infty} \Phi_{p^{*}}\left(\frac{1}{a_{i}} \sum_{k=i}^{\infty} b_{k}\right) .
$$

If

$$
\lim _{m \rightarrow \infty} \sum_{n=1}^{m} \Phi_{p^{*}}\left[\frac{1}{a_{n}} \sum_{j=n}^{m} b_{j}\left[\Phi_{q}\left(\beta_{j+1}\right)\right]^{-1}\right]<\infty,
$$

then (4.18) has solutions in the class $\mathbb{D}_{S}$.

The assumption in Theorem 4.3 (and Corollary 4.4) is not necessary for $\mathbb{D}_{S} \neq \varnothing$ as the following example shows.

Example 4.5. Consider the equation

$$
\Delta^{2} x_{n}=\frac{2}{n(n+1)^{2}(n+2)}\left(x_{n+1}\right)^{-1} .
$$

Clearly, (4.19) is satisfied. We have

$$
\beta_{n}=\sum_{i=n}^{\infty} \sum_{j=i}^{\infty} \frac{2}{j(j+1)^{2}(j+2)}<\sum_{i=n}^{\infty} \sum_{j=i}^{\infty} \frac{2}{j^{4}} .
$$

Taking into account that for $n \in \mathbb{N}, n>1$, and $\gamma$ real positive constant, $\gamma>1$, the following inequality holds

$$
\sum_{i=n}^{\infty} \frac{1}{i^{\gamma}}<\int_{n-1}^{\infty} \frac{1}{x^{\gamma}} d x=\frac{1}{(\gamma-1)(n-1)^{\gamma-1}},
$$

from (4.23) we obtain

$$
\beta_{n+1}<\sum_{i=n+1}^{\infty} \frac{2}{3(i-1)^{3}}=\frac{2}{3} \sum_{i=n}^{\infty} \frac{1}{i^{3}}<\frac{1}{3(n-1)^{2}} .
$$

Hence

$$
\sum_{n=1}^{\infty} \sum_{j=n}^{\infty} b_{j}\left(\beta_{j+1}\right)^{-1}>\sum_{n=1}^{\infty} \sum_{j=n}^{\infty} \frac{6(j-1)^{2}}{j(j+1)^{2}(j+2)}=\infty
$$


and so condition (4.21) is not satisfied. But it is easy to verify that the sequence $\left\{x_{n}\right\}$, $x_{n}=1 / n$, is a solution of $(4.22)$ and $\left\{x_{n}\right\} \in \mathbb{D}_{S}$.

The following result gives an application of Theorem 4.3 to the regular equation (1.3) with the forcing term.

COROLlary 4.6. If $r_{n}>0$ for infinitely many $n$ and

$$
\lim _{m \rightarrow \infty} \sum_{n=1}^{m} \Phi_{p^{*}}\left(\frac{1}{a_{n}} \sum_{k=n}^{m}\left(b_{k}+r_{k}\right)\right)<\infty,
$$

then (1.3) has solutions in the class $\mathbb{D}_{S}$.

Proof. The assertion follows from Theorem 4.3 by choosing $F(n, v)=b_{n}+r_{n}$ and noting that (4.1) is satisfied because $m_{i}=r_{i}>0$.

\section{Concluding remarks}

(1) The continuous case. Decaying solutions of second-order nonlinear singular differential equations without the forcing term have been investigated in $[9,12]$. Corollaries 3.6 and 4.4 can be regarded as the discrete counterparts of [9, Theorem 4.2] and [12, Theorem 5.2], respectively.

(2) An effect of singular nonlinearities. If $Y_{a}=\infty$ and

$$
\lim _{m \rightarrow \infty} \sum_{n=1}^{m} \Phi_{p^{*}}\left(\frac{1}{a_{n}} \sum_{k=n}^{m} b_{k}\right)=\infty
$$

then, from Propositions 3.1 and 4.1, it follows that (4.18) does not possess any decaying solution. This fact cannot occur for equations with regular nonlinearity; for instance, the linear equation

$$
\Delta^{2} x_{n}=(1+n)^{-1} x_{n+1}
$$

has strongly decaying solutions (see, e.g., $[5$, Corollary 3.3(a) $]$ ) and, in this case, $Y_{a}=\infty$ and (5.1) is verified.

(3) An effect of the forcing term $r_{n}$. As we have already noted, (1.3) without the forcing term $r_{n}$ has been investigated in [5]. Comparing the results presented here and in [5], one can see that the existence of regularly decaying solutions of (1.3) remains valid for the equation with the forcing term $r_{n}$ such that $\sum r_{n}<\infty$, while the existence of strongly decaying solutions of (1.3) is caused by the forcing term. More precisely, if (4.19) is satisfied, then (1.3) with $r_{n} \equiv 0$ and $p \leq q$ does not have strongly decaying solutions, see [5, Theorem 2.3]. On the contrary, by Corollary 4.6, (1.3), with the forcing term $r_{n}, r_{n}>0$, for infinitely many $n$, and satisfying (4.27), has strongly decaying solutions.

\section{Acknowledgment}

This work was supported by the Czech Grant Agency, Grant 201/01/0079. 


\section{References}

[1] R. P. Agarwal, Difference Equations and Inequalities. Theory, Methods, and Applications, 2nd ed., Monographs and Textbooks in Pure and Applied Mathematics, vol. 228, Marcel Dekker, New York, 2000.

[2] R. P. Agarwal and D. O’Regan, Singular discrete boundary value problems, Appl. Math. Lett. 12 (1999), no. 4, 127-131.

[3] _ Multiple nonnegative solutions to singular and nonsingular discrete problems, Aequationes Math. 61 (2001), no. 1-2, 97-112.

[4] R. P. Agarwal, D. O’Regan, and P. J. Y. Wong, Positive Solutions of Differential, Difference and Integral Equations, Kluwer Academic Publishers, Dordrecht, 1999.

[5] M. Cecchi, Z. Došlá, and M. Marini, Limit behavior of quasilinear difference equations, to appear in J. Difference Equ. Appl.

[6] - Positive decreasing solutions of quasi-linear difference equations, Comput. Math. Appl. 42 (2001), no. 10-11, 1401-1410.

[7] S. S. Cheng and W. T. Patula, An existence theorem for a nonlinear difference equation, Nonlinear Anal. 20 (1993), no. 3, 193-203.

[8] J. I. Díaz, Nonlinear Partial Differential Equations and Free Boundaries. Vol. I. Elliptic Equations, Research Notes in Mathematics, vol. 106, Pitman (Advanced Publishing Program), Massachusetts, 1985.

[9] T. Kusano and T. Tanigawa, Positive solutions to a class of second order differential equations with singular nonlinearities, Appl. Anal. 69 (1998), no. 3-4, 315-331.

[10] W.-T. Li, X.-L. Fan, and C. Zhong, On unbounded positive solutions of second-order difference equations with a singular nonlinear term, J. Math. Anal. Appl. 246 (2000), no. 1, 80-88.

[11] Positive solutions of discrete Emden-Fowler equation with singular nonlinear term, Dynam. Systems Appl. 9 (2000), no. 2, 247-254.

[12] T. Tanigawa, Asymptotic behavior of positive solutions to nonlinear singular differential equations of second order, Studia Sci. Math. Hungar. 35 (1999), no. 3-4, 427-444.

Mariella Cecchi: Department of Electronics and Telecommunications, University of Florence, Via S. Marta 3, 50139 Florence, Italy

E-mail address: cecchi@det.unifi.it

Zuzana Došlá: Department of Mathematics, Masaryk University, Janáčkovo nám. 2a, 66295 Brno, Czech Republic

E-mail address: dosla@math.muni.cz

Mauro Marini: Department of Electronics and Telecommunications, University of Florence, Via

S. Marta 3, 50139 Florence, Italy

E-mail address: marini@ing.unifi.it 


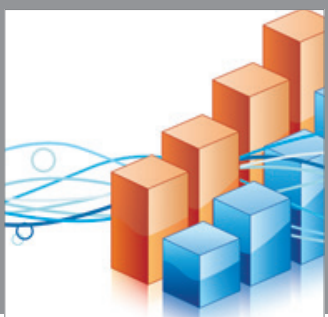

Advances in

Operations Research

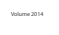

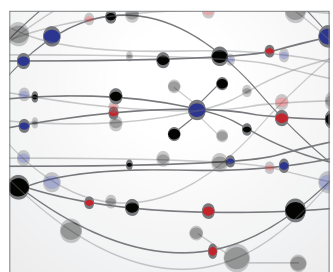

\section{The Scientific} World Journal
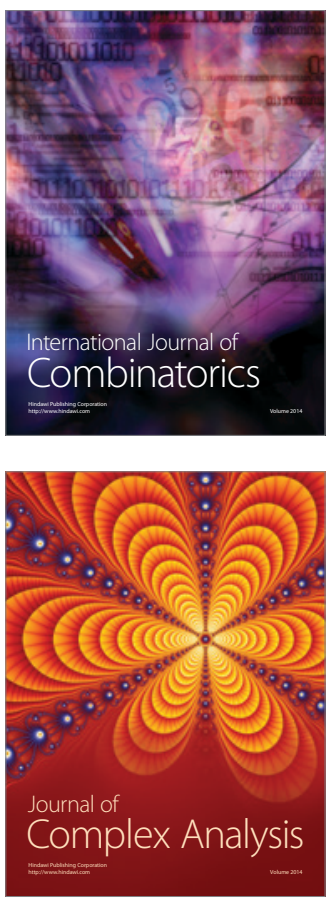

International Journal of

Mathematics and

Mathematical

Sciences
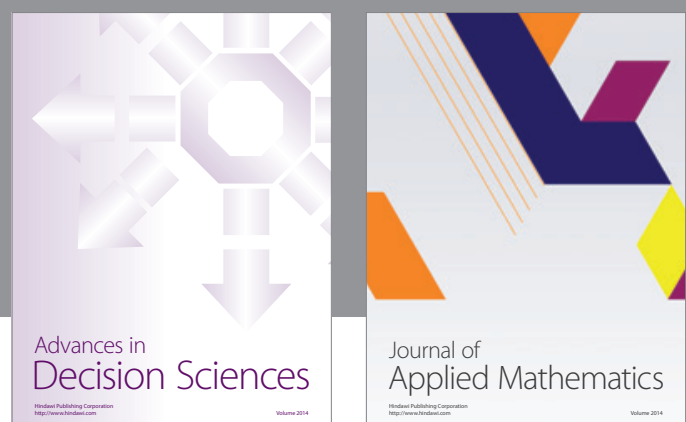

Journal of

Applied Mathematics
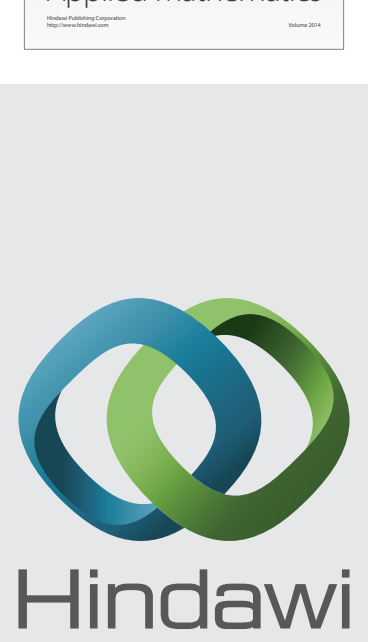

Submit your manuscripts at http://www.hindawi.com
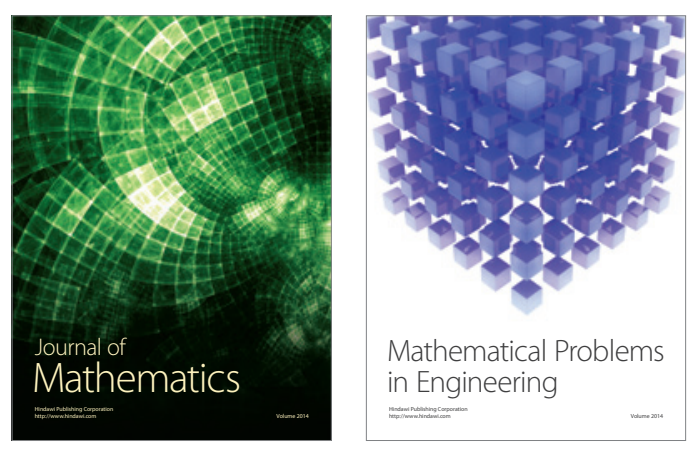

Mathematical Problems in Engineering
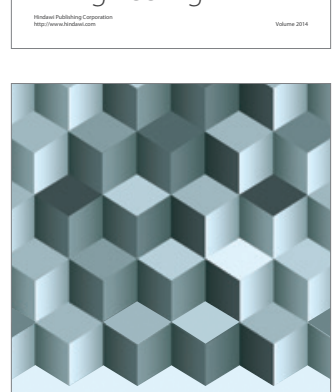

Journal of

Function Spaces
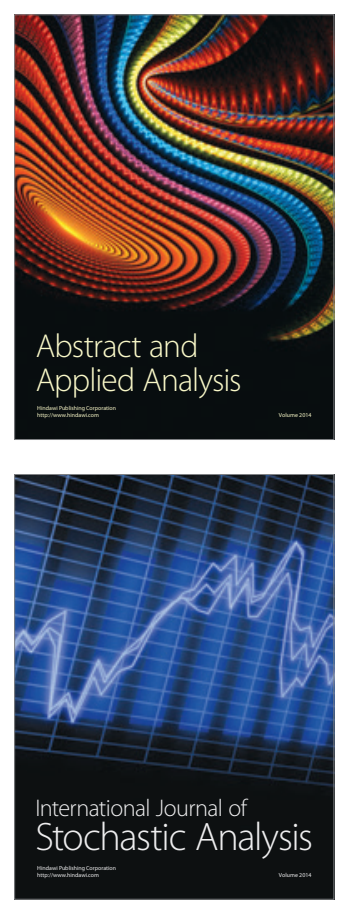

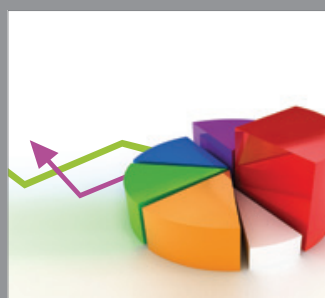

ournal of

Probability and Statistics

Promensencen
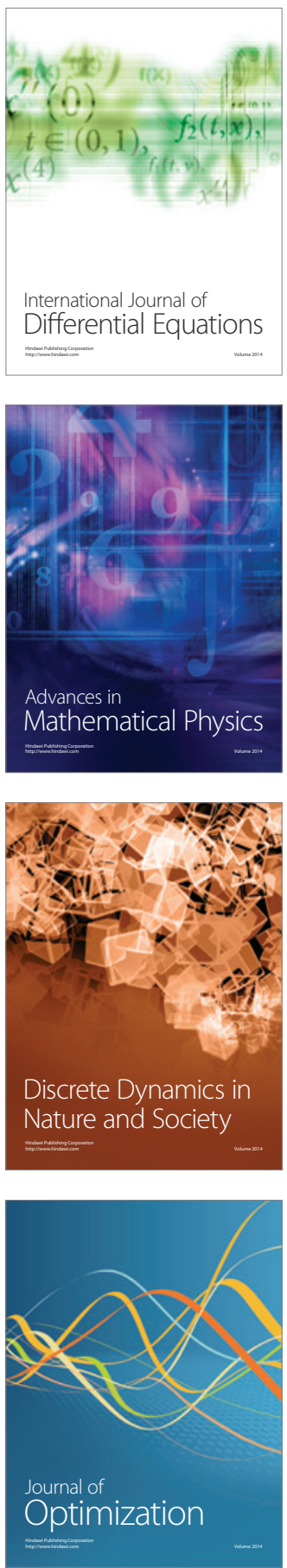